Ocean wave theories

The Dynamics of the Upper Ocean. (Second edition.) By O. M. Phillips. Pp. 336. (Cambridge University: Cambridge, London and New York, 1977.) $£ 16$.

JusT over a decade after its initial publication, Professor Phillips has produced a revised and up-to-date edition of his book. The book remains an applied mathematician's view of ocean surface waves, with an emphasis on the analytical mathematical methods that can be used. It also includes a chapter on internal waves and one on turbulence.

In the ten years before 1966, there had been some exciting developments in our understanding of sea waves. Stimulated initially by military requirements, methods of measuring wave spectra had been developed, and this led to studies of the statistical properties of sea waves and the evolution of the wave field with tiine. Storm waves from half way ro'.. the world could be identified, and useful predictive models of the wave field were being developed. There remained the problems of really understanding the physics of wave growth, the important nonlinear interactions between waves, and the eventual loss of energy from waves by breaking and other mechanisms. But at that time, the solution of these problems could not have seemed too difficult.

The publication of the first edition was timely. It had the merit of covering a rapidly developing field in a uniform and scholarly manner. The mathematical content was also comprehensive and terse, making the book a suitable textbook. The main criticism was that in linking together the established ideas in the subject, the author occasionally slipped in some of his own ideas, which were suspect. However, despite this, the book has been, and is, a well merited success.

The second edition of the book has been extensively revised and a number of small sections added. The first third of the book is concerned with the equations of motion and the basic properties of surface waves. This is the best part of the book, being well written and containing elegant mathematics. New sections on wave action, wave-wave interactions and breaking waves, reflect the importance of these subjects.

- Pulsars by R. N. Manchester and J. H. Taylor (for review, see Nature, 19 January, 271, 285; 1978) has been published by Freeman (Reading) in the UK, price $£ 14.50$.
With these preliminaries out of the way, chapter 4 (which takes up a third of the book) brings together Professor Phillips' ideas on how the sea wave spectra develop. Miles' and Phillips' theories of wave generation are developed at length, as in the first edition of the book, but Banner and Melville's recent observation of enhanced wind drag when a wave breaks is only mentioned briefly. This is surprising as the latter mechanism may well be more important than the traditional theories.

The section on wave-wave interactions is wrong, both in stating that the interactions are local in wave number space and also in seriously applying the analytical results valid for very narrow spectra to a realistic sea spectrum. As a consequence, the author cannot explain the observed enhancement of the peak of the spectrum and gets himself into a bit of a muddle.

Chapter 5 is concerned with internal waves. In the past decade, these have been studied in some detail, stimulated

\section{Ferroelectricity and related topics}

Principles and Applications of Ferroelectrics and Related Materials. By M. E. I ines and A. M. Glass. Pp. xiii + 679. (Oxford University Press/Clarendon: London, Niew York and Oxford, 1977.) $£ 29$.

ONE of the interesting graphs in this book occurs in the preface and shows that the number of publications per year on ferroelectricity and related topics has increased exponentially from one in 1920 to one thousand in 1970 . producing in the process such unlikely terms as "antiferrodistortive" for example, but the authors cannot be held responsible for that, and they successfully convinced me in fact that strontium titanate should not be called an antiferroelectric though ammonium dihydrogen phosphate should. They have been remarkably successful in covering almost every aspect of a subject which now involves most topics in solid-state physics, although they have required 680 pages to do so.

Brief introductory accounts are included of experimental techniques ranging from $X$-ray and neutron crystallography through nuclear and electron paramagnetic resonance to Mossbauer and optical spectroscopy, and of topics as formidable as the renormalisation group procedure in the theory of critical phenomena. Basic concepts are well explained without an excess of mathematical detail. Where the picture remains obscure, by naval interest in the scintillation of sonar signals by internal waves. Garrett and Munk's internal wave spectra are included in the chapter, but the author is more successful at describing the mathematics of the problem than giving physical insight. The last chapter on turbulence is straightforward and contains more on experimental results than the first edition of the book.

Overall, I found the recent development of the subject reflected in this book, a little disappointing. Perhaps the theoreticians are a little too mesmerised by their own analytical methods and perhaps the experimenters were daunted by the cost of studying real sea waves. The subject may have its problems, but the elegant mathematics and coherent viewpoint still make this a useful book.

D. J. Webb

D. J. Webb is a Physical Oceanographer at the Institute of Oceanographic Sciences, Wormley, Godalming, UK.

as in the precise nature of soft modes in materials such as potassium dihydrogen phosphate or the explanation or explanations of the central peak in the energy spectrum of radiation scattered by materials near the critical temperature, there is no attempt to obscure the fact, and though I found a great deal that was new to me, I found very little with which I disagreed. My own prejudices are no doubt responsible for a feeling that the mean field theory is introduced too soon, with the exclusion of any discussion of the special role of coulomb interaction in the lattice dynamics of ionic crystals, but the book is on the whole so comprehensive and successful that it will be a rash author who sets out to write another one on this topic for many years to come.

It is perhaps surprising to find only 65 pages, less than $10 \%$ of the book, devoted to practical applications of ferroelectrics. This is not indicative of any lack of expertise on the part of the authors, hoth of whom are members of staff of Boll Telephone I aboratories, and Cilass has published papers on pyroelectric detectors and electro-optic modulators, for example, but ferroelectrics have never found the range of application of semiconductors, although they are of comparable interest.

It is rare now to find a book of this scope with less than half a dozen authors and a couple of editors. Drs I.ines and Glass have earned our congratulations on both scope and quality.

William Cochran

William Cochran is Professor of Natural Philosophy at the University of Edinburgh, Scotland. 